\title{
Determination of the potential of induced pluripotent stem cells to differentiate into mouse nucleus pulposus cells in vitro
}

\author{
K. Liu', , Z. Chen ${ }^{1,2}$, X.W. Luo ${ }^{2}$, G.Q. Song ${ }^{5}$, P. Wang ${ }^{4}$, X.D. Li ${ }^{3}$, M. Zhao', \\ X.W. Han', Y.G. Bai ${ }^{1,2}$, Z.L. Yang ${ }^{2}$ and G. Feng ${ }^{1,2,3}$ \\ ${ }^{1}$ Institute of Tissue Engineering and Stem Cells, North Sichuan Medical College, \\ Nanchong, Sichuan Province, China \\ ${ }^{2}$ Department of Orthopedic Surgery, Nanchong Central Hospital, Nanchong, \\ Sichuan Province, China \\ ${ }^{3}$ Department of Orthopedic Surgery, University of Virginia School of Medicine, \\ Charlottesville, VA, USA \\ ${ }^{4}$ Department of Pathology, Nanchong Central Hospital, Nanchong, \\ Sichuan Province, China \\ ${ }^{5}$ Department of Biology, North Sichuan Medical College, Nanchong, \\ Sichuan Province, China \\ Corresponding author: G. Feng \\ E-mail: tfg_chb@163.com
}

Genet. Mol. Res. 14 (4): 12394-12405 (2015)

Received April 5, 2015

Accepted July 26, 2015

Published October 14, 2015

DOI http://dx.doi.org/10.4238/2015.October.16.6

ABSTRACT. We determined the potential for induced pluripotent stem (iPS) cells to differentiate into nucleus pulposus (NP)-like cells in mice. iPS cells were generated from tail-tip fibroblasts. We used a pellet culture model with the aim of determining the applicability of iPS cell-based therapy to intervertebral disc degeneration (IVD). The cell pellet was cultured in an NP cell basal medium comprising Dulbecco's modified Eagle's medium supplemented with transforming growth factor beta 1 , dexamethasone, ascorbate-2-phosphate, and 1\% ITS-Premix. The pellet was evaluated by quantitative reverse transcription polymerase chain 
reaction, immunohistochemical staining, and biochemical composition. The differentiation of iPS cells into NP cells was demonstrated by the protein and mRNA expression levels of proteoglycan, collagen II, aggrecan, and CD24. Furthermore, increased hydroxyproline content and dimethylmethylene blue staining demonstrated that the collagen II and glycosaminoglycan content in the NP cells increased with time. We have shown that cultured mouse iPS cells can be induced to differentiate into NP cells. Such proofof-concept opens up the possibility of producing patient-specific NP cells in a relatively simple and straightforward manner with high efficiency. We are confident that such cells could be immediately useful for the study of IVD disease.

Key words: Induced pluripotent stem cells; Nucleus pulposus cells; Intervertebral disc degeneration; Regenerative medicine

\section{INTRODUCTION}

Lower back pain (LBP) is one of the most common musculoskeletal diseases in western countries, with approximately $70-90 \%$ of individuals reporting LBP during their lifetime (Gupta et al., 2012). Although LBP is a multifactorial condition, degeneration of the nucleus pulposus (NP) is thought to be the main cause of intervertebral disc degeneration (IVD) (Ciapetti et al., 2012; Ohta et al., 2012).

Intervertebral discs comprise two main anatomical structures: the annulus fibrosus (AF) and the NP. The AF is an outer layer of fibroblast-like cells arranged in concentric lamellae of collagen fibrils. The NP is surrounded by the AF, and is a gel-like structure that contains chondrocyte-like cells (Bowles et al., 2011; Dahia et al., 2012). During IVD, NP cells decrease in number and the remaining cells have an altered phenotype that is associated with an increased breakdown of the extracellular matrix and altered matrix synthesis (Urban et al., 2004; Skubutyte et al., 2010; Erwin et al., 2011). Traditional treatment options for managing IVD mainly include conservative and surgical treatments. Unfortunately, traditional discectomy cannot restore intervertebral disc function or the spinal column's multi-directional activities (Chiu et al., 2005; Harrop et al., 2008; Masuda and Lotz, 2010). Thus, there is an urgent need to identify means of restoring intervertebral disc function. The NP is the intervertebral disc structure that is most affected by degeneration. Consequently, the focus of many recent studies has been on the use of cell transplantation and tissue engineering strategies for regenerating the NP.

A number of different approaches for regenerating the NP have been described. These include the use of either autologous NP cells (Gruber et al., 2002; Ganey et al., 2003; Meisel et al., 2006; Meisel et al., 2007) or mixtures of bone marrow-derived mesenchymal stem cells (MSCs) and adipose tissue-derived MSCs (Hiyama et al., 2008; Sobajima et al., 2008; Wuertz et al., 2008; Yang et al., 2009; Yang and Li, 2009). Although autologous cells have the advantage of not causing an immune response, it is difficult to obtain the number of cells required for implantation, in contrast to a single disc biopsy. Furthermore, culturing primary NP cells for disc repair is not satisfactory owing to their limited proliferative capacity, which does not allow them to increase to a sufficient number for use in therapy. MSCs offer an alternative with several theoretical advantages over mature cells for cellular therapy. However, the limited number of available MSCs means that there is less differentiation into NP cells. 
Takahashi and Yamanaka (2006) showed for the first time that mouse somatic cells can be reprogrammed to become induced pluripotent stem (iPS) cells via the introduction of a small set of transcription factors that encode OCT3/4, SOX2, KLF4, and c-MYC. These reprogrammed cells exhibit typical embryonic stem (ES) cell colony morphology and demonstrate endogenous expression of pluripotent genes. Subsequent research has demonstrated successful reprogramming of mouse fibroblasts using rats (Li et al., 2009), rhesus monkeys (Liu et al., 2008), and humans (Yu et al., 2007) as the source for generating iPS cell lines. In theory, iPS cell gene therapy can avoid allogeneic immune rejection because the cells are derived from the patient's own cells. iPS cells can also bypass the ethical problems of using human embryos for ES cell line establishment. Differentiated cells generated from patient-derived iPS cells have great potential in regenerative medicine for tissue replacement and cell-based therapies. These cells may also help with drug discovery and/or in elucidating disease pathogenesis (Pietronave and Prat, 2012).

iPS cells can differentiate into various cell types including cardiovascular cells, adipocytes, pancreatic cells, neural cells, and hematopoietic cells (Narazaki et al., 2008; Schenke-Layland et al., 2008; Karumbayaram et al., 2009; Song et al., 2009; Tashiro et al., 2009). Although different methods for the differentiation of ES cells or MSCs into NP cells have been reported, including the use of co-culture systems, growth factors must be added to efficiently induce NP differentiation from ES cells or MSCs. The potential for mouse iPS cells to differentiate into NP-like cells as an alternative has not been fully investigated.

We report the successful induction of the differentiation of mouse pluripotent stem cells into NP-like cells. The addition of transforming growth factor beta 1 (TGF- $\beta 1$ ) efficiently induced mouse pluripotent stem cells to differentiate into functional NP cells. These cells express NP cell lineage markers and have NP functional characteristics.

\section{MATERIAL AND METHODS}

\section{Generation of mouse iPS cells}

iPS cells were generated from tail-tip fibroblasts using the protocol described by Takahashi et al. (2007). Briefly, tail-tip fibroblasts were obtained from BABL/C mice, minced, and cultured in Dulbecco's modified Eagle's medium (DMEM) on a gelatin-coated plate. Cells were cultured and multiplied for six passages before reprogramming occurred. For retroviral particle production, 293T cells were transfected with DNA containing pLentG-KOSM (2 mg) (Cell Biolabs, San Diego, California, USA), pCMV-VSVG (1 mg), and psPAX2 (1.5 mg) (Addgene Cambridge, Massachusetts, USA) using the Fugene HD transfection protocol (Roche, Basel, Switzerland)), according to the manufacturer instructions. The supernatant from the transfected cells was collected and filtered through a $0.22 \mathrm{~mm}$ pore-size filter $48 \mathrm{~h}$ after transfection. The Tail Tip Fibroblasts (TIFs) medium was replaced with virus-containing supernatant supplemented with $8 \mu \mathrm{g} / \mathrm{mL}$ Polybrene (SigmaAldrich, St. Louis, MO, USA). Three days after conducting two transductions, cells were trypsinized and re-plated on irradiated mouse embryonic fibroblasts and cultured in ES medium containing high glucose DMEM, 16\% fetal bovine serum, 1\% L-glutamine, 1-nonessential amino acids, 1 $\mathrm{mM}$ sodium pyruvate, and $100 \mathrm{mM} \beta$-mercaptoethanol (all from Gibco, New York, USA), 1000 U/ $\mathrm{mL}$ murine leukemia inhibitory factor (Chemicon, Billerica, MA, USA), and penicillin/streptomycin (100 and $1000 \mathrm{U}$, respectively) (Gibco, New York). Five days after re-plating infected TIFs, the first ES-like colonies appeared. Individual ES cell-like colonies were monitored for green fluorescent 
protein marker expression during reprogramming. Colonies were handpicked and propagated on irradiated mouse embryonic fibroblasts in ES medium. iPS cell characterizations were performed by investigating stem cell marker expression and teratoma formation.

\section{Karyotyping analysis}

For karyotype analysis, cells were treated with Demecolcine $(0.07 \mathrm{mg} / \mathrm{mL}$, final concentration, Sigma-Aldrich, St. Louis, MO) for $4 \mathrm{~h}$. The cells were subsequently centrifuged and resuspended in $10 \mathrm{~mL} \mathrm{KCl}$ solution $(75 \mathrm{mM})$, followed by three rounds of fixation using an ice-cold methanol: glacial acetic acid (3:1) solution. The fixed cells were washed at least twice with $10 \mathrm{~mL}$ fixative solution before being applied to chilled slides. Slides prepared for chromosome analysis were dried and treated with $0.0025 \%$ trypsin for 5 min and stained with Giemsa (1:10, SigmaAldrich, Louis, MO) for 5-10 min.

\section{Teratoma formation}

iPS cells were collected from mouse embryo fibroblast feeder layers and resuspended at a concentration of $1 \times 10^{7}$ cells $/ \mathrm{mL}$ in DMEM containing $10 \%$ fetal bovine serum. The cell suspension $\left(0.01 \mathrm{~mL} ; 1 \times 10^{6}\right.$ cells) was injected subcutaneously into the flanks of syngeneic mice, and tumors were surgically dissected from the mice 6 weeks later. Tumor samples were embedded in paraffin and the sections were stained with hematoxylin and eosin.

\section{Differentiation culture}

Our main goal was to determine whether the iPS cells that we had generated could be differentiated to an NP cell lineage. We trypsinized $2 \times 10^{5}$ cells and centrifuged them at $500 \mathrm{~g}$ for 10 $\min$ in a $15-\mathrm{mL}$ polypropylene tube. The cell pellet was cultured at $37^{\circ} \mathrm{C}$ in a $5 \% \mathrm{CO}_{2}$ atmosphere in an NP cell basal medium containing DMEM supplemented with TGF- $\beta 1$ (10 ng/mL, Santa Cruz Biotechnology, Santa Cruz, CA, USA), dexamethasone (100 nM, Sigma-Aldrich, Louis, MO), ascorbate2-phosphate $(37.5 \mathrm{mg} / \mathrm{mL})$, and 1\% ITS-Premix (iNPM). The supplements were repeatedly added on alternate days and the media were changed twice per week. Cells cultured in the NP cell media without TGF- $\beta 1$ served as a control group. At Day 21, the pellet was evaluated as described below.

\section{Analysis of mRNA expression levels}

Total RNA from the cell pellet was obtained using the RNeasy Mini kit (Qiagen, Valencia, CA, USA). The RNA was reverse transcribed to complementary DNA (cDNA) using the First Strand cDNA kit (Promega, Madison, WI, USA) following the manufacturer protocol. Quantitative polymerase chain reaction (qPCR) analysis was then performed using the Quantitect SYBR Green PCR master mix (Qiagen, Valencia, CA). Standard curves were generated, and quantities of each transcript were normalized to glyceraldehyde 3-phosphate dehydrogenase as an internal control.

\section{Histology analysis}

The pellet cultures were harvested, washed with phosphate-buffered saline to remove the medium, and fixed in $4 \%$ paraformaldehyde for $4 \mathrm{~h}$. The samples were then dehydrated by 
treatment with a graded series of alcohol concentrations, and embedded in paraffin. Sections were cut to $5-\mu m$ thicknesses and stained with Safranin-O/ Fast Green, and hematoxylin and eosin.

\section{Immunohistochemical staining}

Immunochemical staining for collagen II was performed using a collagen staining kit (Chondrex, Redmond, WA, USA), with minor modifications to the manufacturer instructions. The paraffin-embedded sections were deparaffinized and endogenous peroxidase activity was quenched with $3 \%$ hydrogen peroxide in methanol for $10 \mathrm{~min}$ at room temperature. Sections were treated with $2 \%$ bovine testicular hyaluronidase for $30 \mathrm{~min}$ at $25^{\circ} \mathrm{C}$, and then incubated with blocking buffer for 30 min, followed by exposure to Arthrogen-CIA collagen II monoclonal antibodies at a dilution of 1:250 overnight at $4^{\circ} \mathrm{C}$. Streptavidin peroxidase $(50 \mathrm{~mL}$ in $10 \mathrm{~mL}$ streptavidin peroxidase-dilution buffer) was applied for $1 \mathrm{~h}$ at room temperature. Sections were then developed with 3,3'-diaminobenzidine for $30 \mathrm{~min}$. For visualization of cluster of differentiation 24 (CD24) protein and aggrecan, sections were treated as above, blocked with goat $\lg G(1: 250)$ for $2 \mathrm{~h}$ at room temperature, then incubated with either rabbit anti-mouse aggrecan polyclonal antibody $(5 \mathrm{mg} / \mathrm{mL}$, Santa Cruz Biotechnology, Santa Cruz, CA) or rat anti-mouse CD24 polyclonal antibody $(1 \mathrm{mg} / \mathrm{mL}$, Santa Cruz Biotechnology, Santa Cruz, CA) for $12-18 \mathrm{~h}$ at $4^{\circ} \mathrm{C}$, followed by incubation with Texas red- (for CD24) or fluorescein isothiocyanate- (for aggrecan) conjugated secondary antibody for $1 \mathrm{~h}$ at room temperature.

\section{Biochemical composition}

To determine the biochemical composition of the cultured NP cells, a total of 60 samples were digested in $200 \mu \mathrm{L}$ sterile papain solution $(125 \mathrm{~g} / \mathrm{mL}$ in 1 x PBE, pH 6.5; PBE is phosphatebuffered saline with ethylenediaminetetraacetic acid) at $60^{\circ} \mathrm{C}$ for $18 \mathrm{~h}$. Total DNA content of the pellets was determined using the Hoechst 33258 (Sigma-Aldrich, Louis, MO) fluorometric dye assay with calf thymus DNA as a standard. The sulfated glycosaminoglycan (sGAG, Sigma-Aldrich, Louis, MO) content of the samples was measured in a modified dimethylmethylene blue colorimetric assay (21 mg 1,9-dimethylmethylene blue in $5 \mathrm{~mL}$ absolute ethanol and $2.0 \mathrm{~g}$ sodium formate, which was volume-adjusted to $800 \mathrm{~mL}$ with distilled water and $\mathrm{pH}$-adjusted to 1.5 with formic acid), using chondroitin sulfate as the standard. The hydroxyproline (hypro) content was determined after hydrolysis of the tissue in $6 \mathrm{~N} \mathrm{HCl}$ for $24 \mathrm{~h}$ at $110^{\circ} \mathrm{C}$ using the dimethylaminobenzaldehyde (SigmaAldrich, Louis, MO) colorimetric assay. For each assay, all pellets and standards were processed in duplicate as part of a single experiment and repeated at least twice to confirm the results. The following ratios were calculated for each group: sGAG/DNA and hypro/DNA ratio.

\section{Statistical analysis}

Statistical evaluation was performed using analysis of variance followed by a post hoc Student's $t$-test. AP value less than 0.05 was considered significant. Data are expressed as mean \pm SD.

\section{RESULTS}

\section{Generation of mouse iPS cells using a polycistronic lentiviral expression vector}

We introduced lentiviral expression vectors containing Oct3/4, Sox2, Klf4, and c-Myc 
into the somatic cells, which were transferred to feeder layers following the second transduction. ES cell-like cell colonies appeared from the infected cells 5 days after viral infection. Ten single colonies were picked on Day 15 after viral infection and expanded to establish iPS cell lines. One mouse iPS cell line was selected for utilization in this study and maintained successfully for over 30 passages; it was characterized by round, multilayer colonies having clearly defined edges, and resembled the typical colony morphology of mouse ES cells (Figure 1A).

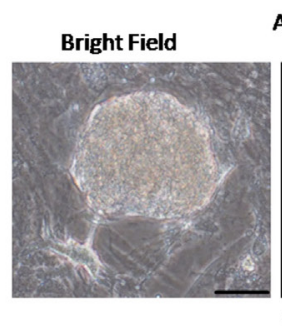

A

GFP

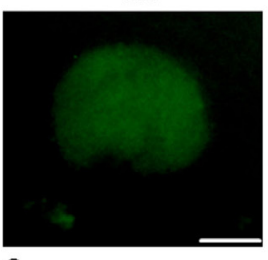

c

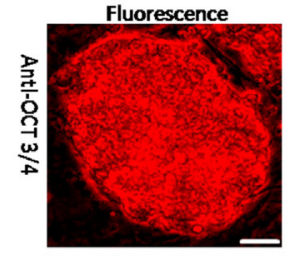

Bright Field

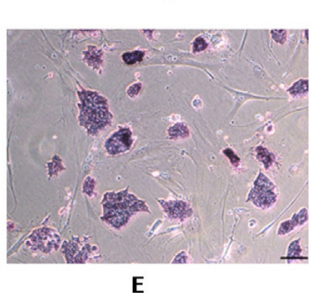

$\mathbf{E}$
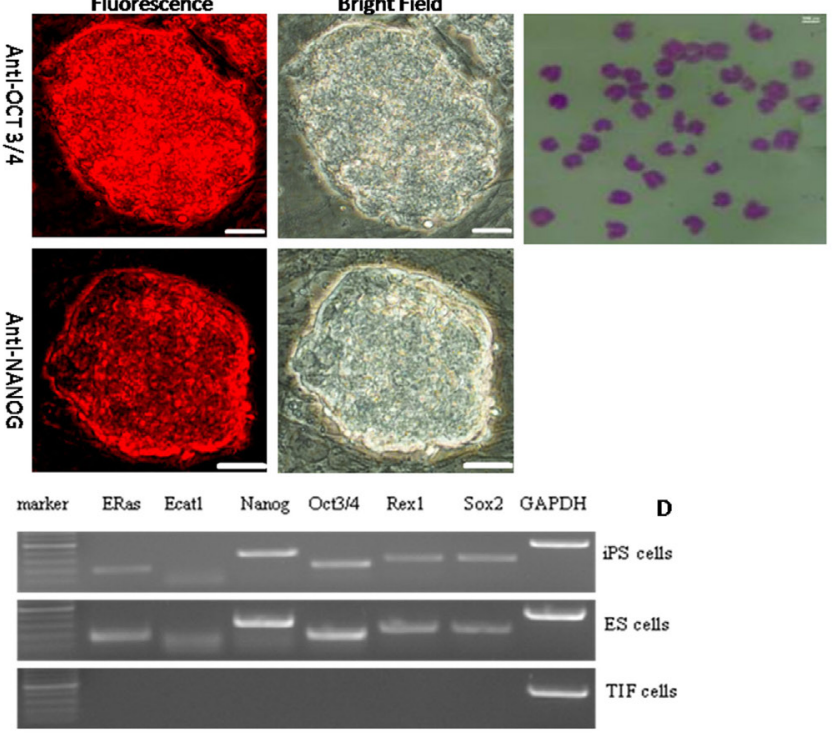

D

F
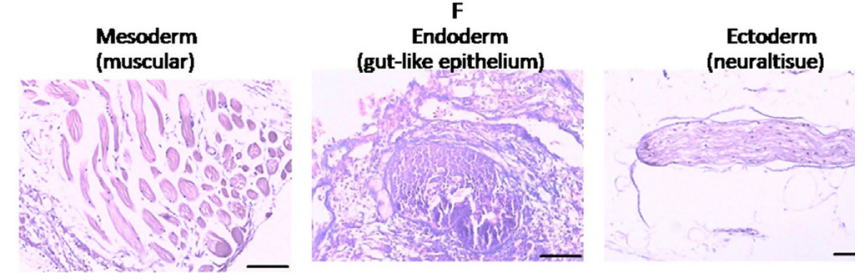

Figure 1. Characterization of induced pluripotent stem (iPS) cell colonies generated from mouse embryonic fibroblasts (MEFs). A. Fluorescence images of green fluorescent protein (GFP) during reprogramming (magnification 200X, bar $=50 \mu \mathrm{m}$ ). B. Alkaline phosphatase (AP) staining (magnification 100X, bar $=100 \mu \mathrm{m}$ ) in induced embryonic stem (ES) cell-like cell colonies. C. Staining of pluripotency markers (OCT3/4 and NANOG) in induced cell colonies (magnification 200X, bar $=25 \mu \mathrm{m})$. D. Reverse transcription polymerase chain reaction (RT-PCR) analysis of pluripotency marker gene expression in induced colonies. E. Karyotyping analysis of a representative colony (magnification 1000X). F. Hematoxylin and eosin (HE) staining of teratomas derived from iPS cells (magnification 100X, bar $=100 \mu \mathrm{m}$ ). 


\section{Characterization of mouse iPS cells}

Alkaline phosphatase activity staining was performed to identify iPS cell lines that were positive for alkaline phosphatase (Figure 1B). To study these ES cell-like colonies in more detail, immunofluorescence staining was performed using different established ES cell markers (NANOG and OCT3/4). Positive red immunofluorescence was observed in the nuclei of iPS cells expressing NANOG and OCT3/4 (Figure 1C). In addition, we performed reverse transcription PCR (RT-PCR) to determine whether ES cell marker genes were expressed in iPS cells. We used primers that would amplify transcripts of the endogenous gene but not transcripts of the transgene. Gene expression analysis showed that mouse iPS cells expressed many undifferentiated ES cell-marker genes (Oct3/4, Sox2, Nanog, Rex1, Eras, and Ecat1) at levels equivalent to or higher than those in the mouse ES cell lines (Figure 1D). Further analysis revealed that the iPS cells from this clone were karyotypically normal (Figure 1E).

To further test the pluripotency of iPS cells in vivo, we injected iPS cells subcutaneously into the flanks of syngeneic BABL/C mice, and teratomas containing iPS cells became palpable 3 weeks after injection. Teratomas were removed and histologically examined 6 weeks after injection. The teratomas contained tissues derived from three embryonic germ layers: neural tissues (ectoderm), muscular tissues (mesoderm), and gut-like epithelium (endoderm). Overall, these results indicate that the iPS cells have full capacity to differentiate into the three germ layers in vivo (Figure 1F).

\section{Glycosaminoglycan (GAG) and hypro}

iPS cells were cultured in a pellet system and treated with $10 \mathrm{ng} / \mathrm{mL}$ TGF- $\beta 1$ to determine if they could be induced into NP cells. Negative control pellets were maintained in basic culture media in the absence of growth factors.

To verify the production of an NP matrix by iPS cells, GAG was measured to estimate the total proteoglycan within the cell pellets, whereas hypro was used to estimate the total collagen content. Both GAG and hypro were normalized to DNA content. Synthesis of sGAG by pellets cultured in basic culture media was low after 1 week, and did not significantly increase over time. Treatment with TGF- $\beta 1(100 \mathrm{ng} / \mathrm{mL})$ induced synthesis of sGAG more than in the negative controls, and levels of SGAG gradually increased during each sampling period $(P<0.05)$ (Figure $2 A)$, as did total intracellular collagen (Figure $2 B$ ). GAG was measured to estimate the total proteoglycan within the disc, whereas hypro was used to estimate the total collagen content.
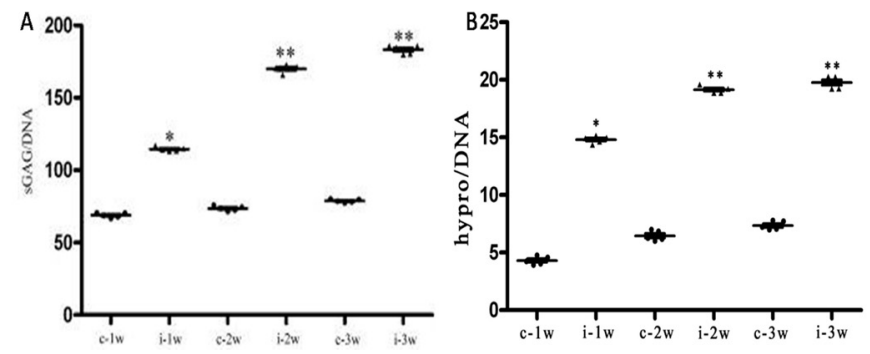

Figure 2. Biochemical analysis of high density, pellet-cultured induced pluripotent stem (iPS) cells after induction of nucleus pulposus (NP)-like cells. A. Synthesis of sulfated glycosaminoglycan (sGAG) from iPS cells cultivated within transforming growth factor beta 1 (TGF- $\beta 1$ ) medium. B. Synthesis of total intracellular collagen from iPS cells cultivated in TGF- $\beta 1$ medium. Values are mean $\pm \mathrm{SD}, \mathrm{N}=3$. ${ }^{*} \mathrm{P}<0.05$ vs control medium $(\mathrm{CM})$ group. ${ }^{* *} \mathrm{P}<0.01$ vs $\mathrm{CM}$ group. 


\section{Gene expression of iPS cells cultured in TGF- $\beta 1$ medium}

Quantitative PCR was used to measure the gene expression profile of NP cells induced by TGF- $\beta 1$ and levels of RNA transcripts for collagen II and aggrecan. Compared with the basic culture media, TGF- $\beta 1$ treatments strongly induced expression of collagen II and aggrecan. After 1 week in culture, the TGF- $\beta 1$-treated group had higher collagen II expression levels than pellets maintained in basic culture $(P<0.05)$. At 1 week, both collagen II and aggrecan mRNA levels were significantly higher for the TGF- $\beta 1$ groups, with expression peaking at this time point. At 3 weeks, collagen II and aggrecan expression in the growth factor-treated group decreased compared with levels at 2 weeks, but levels were still higher than in the controls (Figure 3). These data demonstrate that iPS cells can be induced to differentiate to NP cells under TGF- $\beta 1$ treatment.
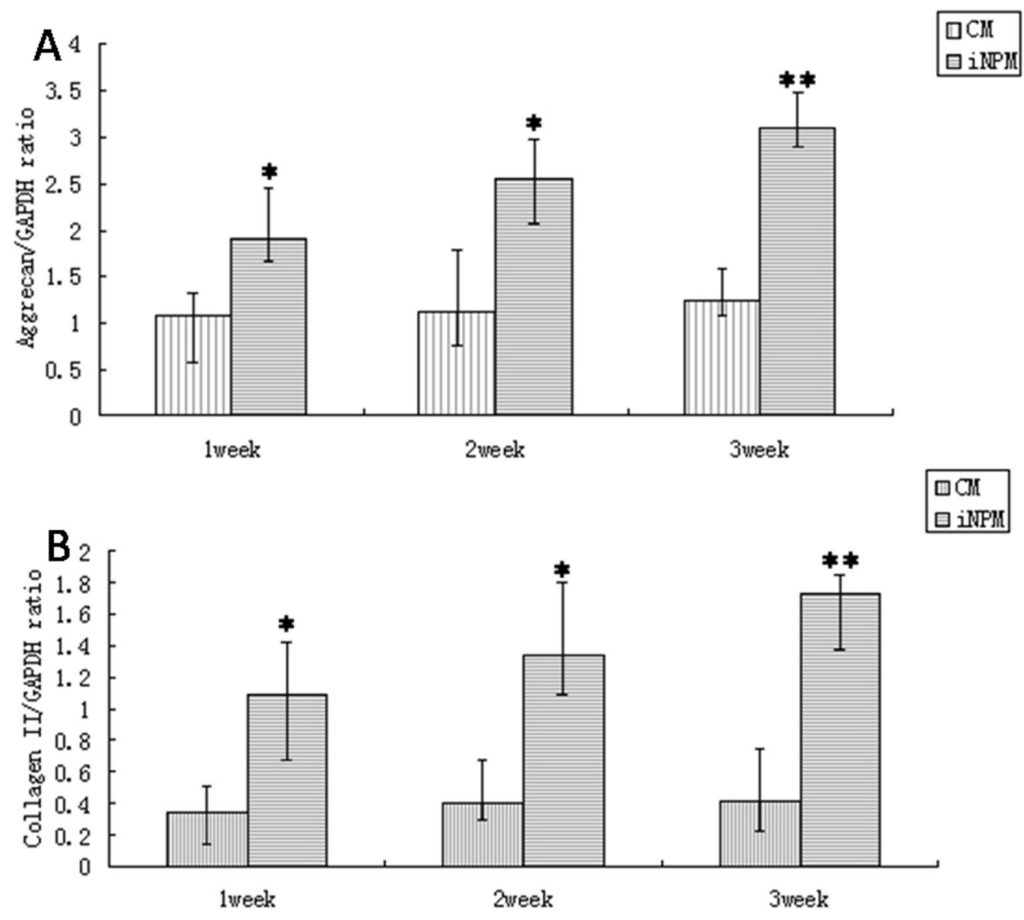

Figure 3. Gene expression patterns of high-density, micromass-cultured induced pluripotent stem (iPS) cells after induction of nucleus pulposus (NP) cells over 1, 2, or 3 weeks. A. mRNA for aggrecan. B. mRNA for collagen II. Data are presented as mean $\pm \mathrm{SD}(\mathrm{N}=6) .{ }^{*} \mathrm{P}<0.05$ vs control medium $(\mathrm{CM})$ group. ${ }^{* *} \mathrm{P}<0.01$ vs $\mathrm{CM}$ group.

\section{Histological and immunohistochemistry analysis of iPS cells cultured in TGF- $\beta 1$ medium}

Pellet cultures were histologically assessed with Safranin-O staining to visualize deposition of a proteoglycan-rich extracellular matrix. TGF- $\beta 1$ increased deposition of proteoglycans compared with the basic culture (Figure 4A). Additional pellets were harvested and immunostained for collagen II (immunochemical staining), aggrecan, and CD24 (all by immunofluorescence staining). 

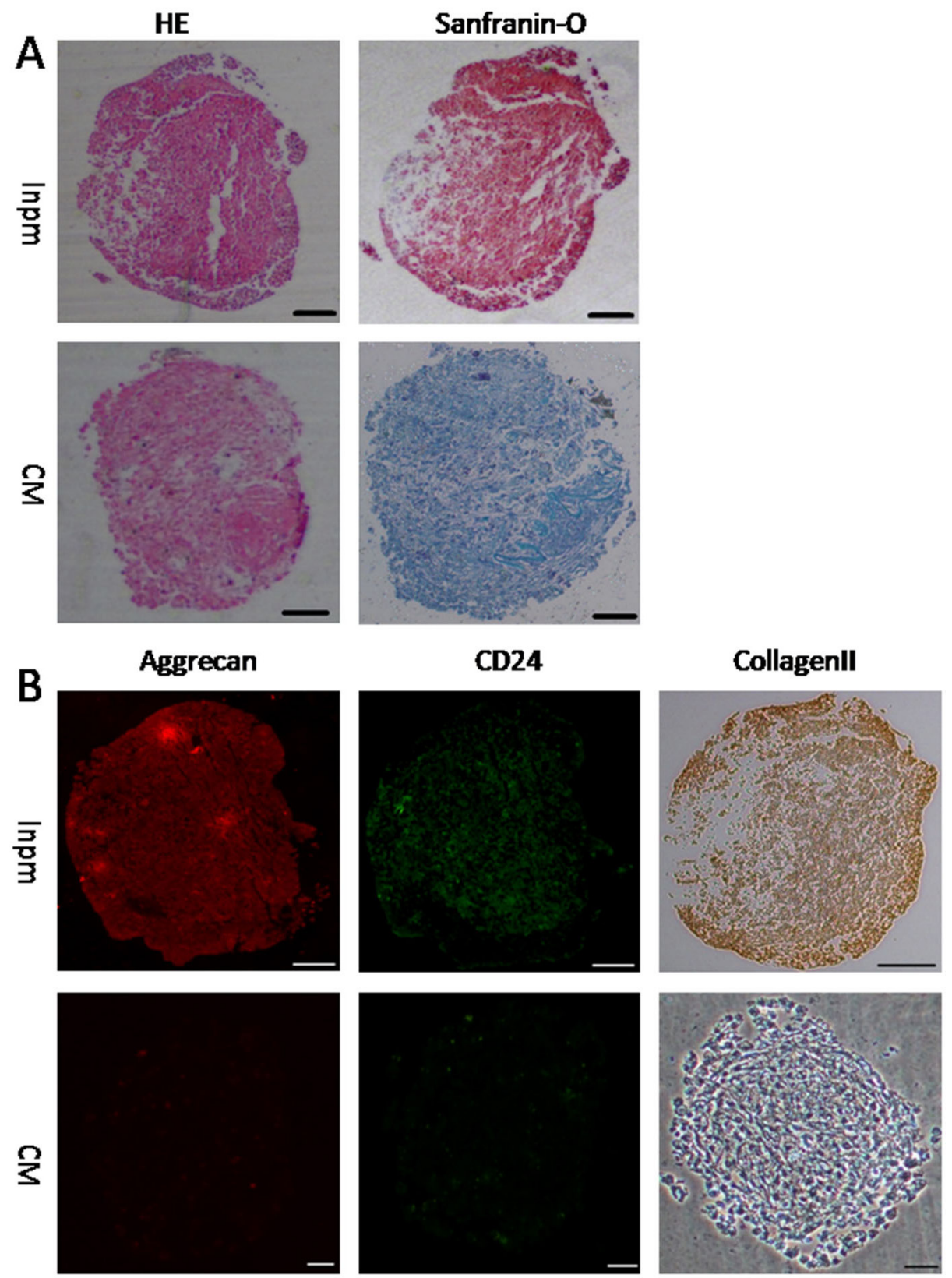

Figure 4. A. Safranin-O and hematoxylin and eosin (HE) staining of proteoglycan-rich extracellular matrix in representative induced pluripotent stem (iPS) cell pellets (magnification 100X, bar $=100 \mu \mathrm{m}$ ). B. Immunostaining of iPS cell pellet cultures for the presence of collagen II, aggrecan, and cluster of differentiation 24 (CD24) in iPS cells following treatment with transforming growth factor beta 1 (TGF- $\beta 1$ ) for 3 weeks. Regions of positive staining are shown by green fluorescence (CD24), dark brown (collagen II), or red fluorescence (aggrecan) (magnification 100X, bar $=100 \mu \mathrm{m})$. 
Pellets cultured in basic culture in the presence of TGF- $\beta 1$ demonstrated robust staining for collagen II, aggrecan, and CD24. Typically, staining intensity was higher towards the periphery and lower in the central regions of pellets cultured in the presence of TGF- $\beta 1$. iPS cell pellets cultured in basic culture showed weaker staining for collagen II, aggrecan, and CD24 compared to the TGF- $\beta 1$ treatment groups. This, along with the biochemical data and Safranin-O staining, clearly demonstrates that NP cell transformation of iPS cells occurred at an enhanced rate in the presence of growth factor induction (Figure 4B).

\section{DISCUSSION}

Several studies have described the differentiation of ES cells or MSCs into cells that display NP characteristics (Sheikh et al., 2009). However, the risk of immune rejection and ethical criticisms have made work with ES cells less promising. The recent breakthrough in iPS cell technology demonstrates that iPS-derived cells may be an alternative to ES-based methods. This study is the first to report the successful induction of mouse pluripotent stem cells into functional NP cells using TGF $\beta 1$ and a simple, efficient process.

Recently, numerous articles have demonstrated that the reprogramming of somatic cells to a pluripotent state can be achieved by forced expression of several transcription factors through diverse genetic modification methods, and that the resulting induced pluripotent stem cells resemble ES cells in many ways. Although teratoma and viable chimera formation have proved the pluripotency of iPS cells, there are few studies discussing the potential of the in vitro differentiation of iPS cells into specific cell lineages. Therefore, the present study aimed to investigate the NP differentiation potential of a mouse iPS cell clone.

Multiple cytokines and growth factors, such as members of the TGF- $\beta$ and bone morphogenetic protein families, insulin-like growth factor-1, and fibroblast growth factor-2 (Ehlicke et al., 2010; Luo et al., 2011), promote NP cells from ES cells or MSCs. Many of these cytokines and growth factors display a high degree of functional overlap. In previous studies, we have demonstrated that adipose tissue-derived mesenchymal stem cells express collagen II and aggrecan genes, and increase protein synthesis when cultured with TGF- $\beta 1(10 \mathrm{ng} / \mathrm{mL}) \mathrm{or}$ GDF (100 ng/mL) (Feng et al., 2008). Based on our protocol, mouse iPS cells were efficiently differentiated into NP-like cells. These iPS cell-derived, NP-like cells had features typical of NP cells, such as expression of collagen and aggrecan, expression of other NP markers, CD24 protein synthesis, and functional NP characteristics (e.g., glycosaminoglycan storage). Consequently, these cells appear to be fully functional in vitro, and may serve as a model for IVD-related research and potential practical applications.

Proof that mouse iPS cells can be differentiated into functional NP cells is an encouraging result for human iPS research and therapy. The major advantages of iPS technology over ES cells include bypassing immune system rejection by use of autologous NPs. However, several issues must be resolved before iPS-derived cells can be safely applied in clinical settings. One issue is the potential risk of contaminating cultures with undifferentiated iPS cells. Furthermore, for potential clinical application, iPS cells without any genomic modifications should be used. This means that non-viral, non-integrative iPS induction protocols should be further developed and optimized. Consequently, the latest developments using proteins for iPS reprogramming are very promising. Finally, the entire iPS induction and differentiation procedure would need to be established under fully defined, xeno-free conditions. 
In summary, we have shown that cultured mouse iPS cells can be induced to produce differentiated NP cells. We acknowledge that compared to the in vivo IVD environment, artificial culture conditions are more likely to affect the function of iPS-derived NP cells. Nevertheless, our data demonstrate the feasibility of generating cells with NP characteristics using an iPS cell intermediate. Such proof-of-concept opens up the possibility of producing patient-specific NP in a relatively simple and straightforward manner with high efficiency. We are confident that such cells could be immediately useful for the study of IVD.

\section{Conflicts of interest}

The authors declare no conflict of interest.

\section{ACKNOWLEDGMENTS}

Research supported by grants from the National Natural Science Foundation of China (\#81171472, \#81071270, and \#30872614), the Sichuan Provincial Science and Technology Support Program (\#2010SZ0048, \#2008SZ0103), and the Sichuan Province Outstanding Youth Leader Grant Program (\#2009-05-396).

\section{REFERENCES}

Bowles RD, Gebhard HH, Härtl R and Bonassar LJ (2011). Tissue-engineered intervertebral discs produce new matrix, maintain disc height, and restore biomechanical function to the rodent spine. Proc. Natl. Acad. Sci. U S A. 108: 13106-13111.

Chiu JC, Clifford T, Princenthal R and Shaw S (2005). Junctional disc herniation syndrome in post spinal fusion treated with endoscopic spine surgery. Surg. Technol. Int. 14: 305-315.

Ciapetti G, Granchi D, Devescovi V, Leonardi E, et al. (2012). Ex vivo observation of human intervertebral disc tissue and cells isolated from degenerated intervertebral discs. Eur. Spine J. 21: S10-S19.

Dahia CL, Mahoney E and Wylie C (2012). Shh signaling from the nucleus pulposus is required for the postnatal growth and differentiation of the mouse intervertebral disc. PLoS One 7: e35944.

Ehlicke F, Freimark D, Heil B, Dorresteijn A, et al. (2010). Intervertebral disc regeneration: influence of growth factors on differentiation of human mesenchymal stem cells (hMSC). Int. J. Artif. Organs 33: 244-252.

Erwin WM, Islam D, Inman RD, Fehlings MG, et al. (2011). Notochordal cells protect nucleus pulposus cells from degradation and apoptosis: implications for the mechanisms of intervertebral disc degeneration. Arthritis Res. Ther. $13:$ R215.

Feng G, Wan Y, Balian G, Laurencin CT, et al. (2008). Adenovirus-mediated expression of growth and differentiation factor-5 promotes chondrogenesis of adipose stem cells. Growth Factors 26: 132-142.

Ganey T, Libera J, Moos V, Alasevic O, et al. (2003). Disc chondrocyte transplantation in a canine model: a treatment for degenerated or damaged intervertebral disc. Spine 28: 2609-2620.

Gruber HE, Johnson TL, Leslie K, Ingram JA, et al. (2002). Autologous intervertebral disc cell implantation: a model using Psammomys obesus, the sand rat. Spine 27: 1626-1633.

Gupta G, Radhakrishna M, Chankowsky J and Asenjo JF (2012). Methylene blue in the treatment of discogenic low back pain. Pain Phys. 15: 333-338.

Harrop JS, Youssef JA, Maltenfort M, Vorwald P, et al. (2008). Lumbar adjacent segment degeneration and disease after arthrodesis and total disc arthroplasty. Spine 33: 1701-1707.

Hiyama A, Mochida J, Iwashina T, Omi H, et al. (2008). Transplantation of mesenchymal stem cells in a canine disc degeneration model. J. Orthop. Res. 26: 589-600.

Karumbayaram S, Novitch BG, Patterson M, Umbach JA, et al. (2009). Directed differentiation of human-induced pluripotent stem cells generates active motor neurons. Stem Cells 27: 806-811.

Li W, Wei W, Zhu S, Zhu J, et al. (2009). Generation of rat and human induced pluripotent stem cells by combining genetic reprogramming and chemical inhibitors. Cell Stem Cell 4: 16-19.

Liu H, Zhu F, Yong J, Zhang P, et al. (2008). Generation of induced pluripotent stem cells from adult rhesus monkey fibroblasts. Cell Stem Cell 3: 587-590. 
Luo W, Xiong W, Quu M, Lv Y, et al. (2011). Differentiation of mesenchymal stem cells towards a nucleus pulposus-like phenotype utilizing simulated microgravity in vitro. J. Huazhong Univ. Sci. Technol. Med. Sci. 31: 199-203.

Masuda K and Lotz JC (2010). New challenges for intervertebral disc treatment using regenerative medicine. Tissue Eng. Part B Rev. 16: 147-158.

Meisel HJ, Ganey T, Hutton WC, Libera J, et al. (2006). Clinical experience in cell-based therapeutics: intervention and outcome. Eur. Spine J. 15: S397-S405.

Meisel HJ, Siodla V, Ganey T, Minkus Y, et al. (2007). Clinical experience in cell-based therapeutics: disc chondrocyte transplantation A treatment for degenerated or damaged intervertebral disc. Biomol. Eng. 24: 5-21.

Narazaki G, Uosaki H, Teranishi M, Okita K, et al. (2008). Directed and systematic differentiation of cardiovascular cells from mouse induced pluripotent stem cells. Circulation 118: 498-506.

Ohta R, Tanaka N, Nakanishi K, Kamei N, et al. (2012). Heme oxygenase-1 modulates degeneration of the intervertebral disc after puncture in Bach 1 deficient mice. Eur. Spine J. 21: 1748-1757.

Pietronave S and Prat M (2012). Advances and applications of induced pluripotent stem cells. Can. J. Physiol. Pharmacol. 90: 317-325.

Schenke-Layland K, Rhodes KE, Angelis E, Butylkova Y, et al. (2008). Reprogrammed mouse fibroblasts differentiate into cells of the cardiovascular and hematopoietic lineages. Stem Cells 26: 1537-1546.

Sheikh H, Zakharian K, De La Torre RP, Facek C, et al. (2009). In vivo intervertebral disc regeneration using stem cell-derived chondroprogenitors. J. Neurosurg. Spine 10: 265-272.

Skubutyte R, Markova D, Freeman TA, Anderson DG, et al. (2010). Hypoxia-inducible factor regulation of ANK expression in nucleus pulposus cells: possible implications in controlling dystrophic mineralization in the intervertebral disc. Arthritis Rheum. 62: 2707-2715.

Sobajima S, Vadala G, Shimer A, Kim JS, et al. (2008). Feasibility of a stem cell therapy for intervertebral disc degeneration. Spine J. 8: 888-896.

Song Z, Cai J, Liu Y, Zhao D, et al. (2009). Efficient generation of hepatocyte-like cells from human induced pluripotent stem cells. Cell Res. 19: 1233-1242.

Takahashi K and Yamanaka S (2006). Induction of pluripotent stem cells from mouse embryonic and adult fibroblast cultures by defined factors. Cell 126: 663-676.

Takahashi K, Okita K, Nakagawa M and Yamanaka S (2007). Induction of pluripotent stem cells from fibroblast cultures. Nat. Protoc. 2: 3081-3089.

Tashiro K, Inamura M, Kawabata K, Sakurai F, et al. (2009). Efficient adipocyte and osteoblast differentiation from mouse induced pluripotent stem cells by adenoviral transduction. Stem Cells 27: 1802-1811.

Urban JP, Smith S and Fairbank JC (2004). Nutrition of the intervertebral disc. Spine 29: 2700-2709.

Wuertz K, Godburn K, Neidlinger-Wilke C, Urban J, et al. (2008). Behavior of mesenchymal stem cells in the chemical microenvironment of the intervertebral disc. Spine 33: 1843-1849.

Yang F, Leung VY, Luk KD, Chan D, et al. (2009). Mesenchymal stem cells arrest intervertebral disc degeneration through chondrocytic differentiation and stimulation of endogenous cells. Mol. Ther. 17: 1959-1966.

Yang $X$ and Li X (2009). Nucleus pulposus tissue engineering: a brief review. Eur. Spine J. 18: 1564-1572.

Yu J, Vodyanik MA, Smuga-Otto K, Antosiewicz-Bourget J, et al. (2007). Induced pluripotent stem cell lines derived from human somatic cells. Science 318: 1917-1920. 